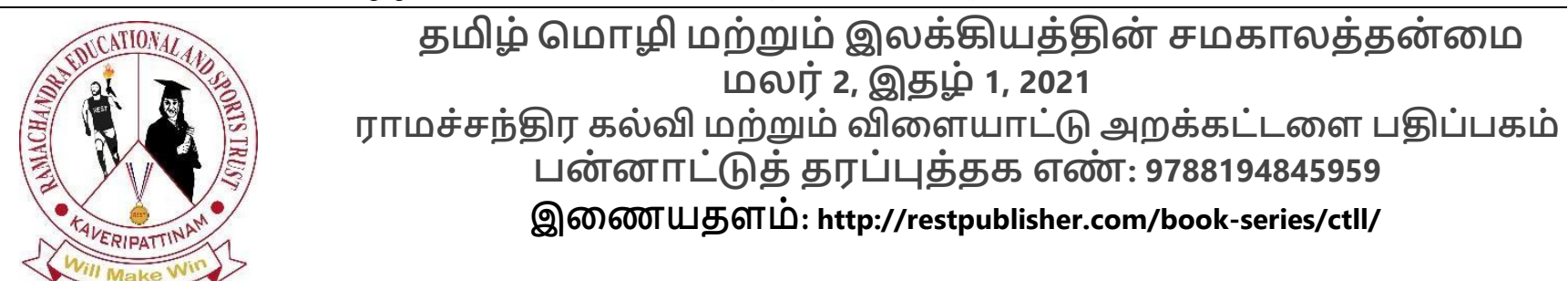

\title{
பதினெண் கீழ்க்கணக்கு நூல்களில் பன்முகச் சிந்தனை
}

\author{
முனைவர் சூ·ஆரோக்கியமோி \\ உதவிப்பேராசிரியர், அருள் ஆனந்தர் கல்லூரி, கருமாத்தூர்.
}

இலக்கியத்தின் பொற்காலம் எனப் போற்றப்பெற்ற சங்கஇலக்கியம் என்னும் பதினெண்மேற்கணக்கு நூல்களைத் தொடர்ந்து தமிழில் நீதிகளையும் நெறிமுறைகளையும் எடுத்தோதுவதற்குப் பதினெட்டு வகை நூல்கள் தோன்றின. சங்ககாலத்தின் இறுதிப் பகுதி ஆடம்பரமும் ஆரவாரமும் நிறைந்திருந்தது. கலை என்ற பெயரில் ஒழுக்கக் கேடுகள் பெருகின. சமுதாய நிலையில் வாணிபப் பொருளாதாரத் தீய விளைவுகள் ஏற்றத்தாழ்வுகள் உழைப்பின்றி வாழும் தன்மை உருவாயின. காதல் வீரம் கொடை முதலிய சிறந்த உணர்ச்சிகளிலும் இயற்கை இன்பத்திலும் ஈடுபட்டிருந்த புலவர்களிகன் உள்ளம் அடுத்த சில தலைமுறைகளில் நீதிகளைப் பாடும் நிலைக்கு மாறியது. அந்தகாலத்தைச் சங்கமருவிய காலம் அல்லது நீதிநூல் காலம் என்பர். அப்போது பழைய சோ சோழ பாண்டியரின் அமைதியான ஆட்சியில் கலகம் நேர்ந்தது என்றும் களப்பிரர் என்னும் சிலர் பகுந்து நாட்டு மக்களின் அமைதிமைக் கெடுத்தார்கள் என்றும் வரலாற்றில் செய்தி உள்ளது. ஆகவே சமுதாயத்தில் நீதியைப் போதிக்க வேண்டிய கடமை புலவர்களுக்கு நேர்ந்தது. அப்படிப்பட்ட சூழ்நிலையில் தோன்றியதே பதினெண் கீழ்க்கணக்கு நூல்களாகும்.

அறநூல்களின் வளர்ச்சி நிலை

தமிழ்ப்புலவர்கள் காதலையும் வீரத்தையும் இரு கண்களாகவும் அறத்தை உயிராகவும் மதித்தனர். ஆதனால் தாம் பாடியபாடல்களில் அறக்கருத்துக்களை வாய்ப்பு நேரும் போதெல்லாம் வலியுறுத்தினர்கள். அவர்களுக்குப் பின்னால் வந்தவர்கள் இக்கருத்துக்களை

விளக்க தனியாகவே அறநூல்களைஇயற்றினர். ஆதற்குக் காரணம் சமூக மாற்றமாக இருக்கலாம். சுங்க காலத்தில் திணை அமைப்பு முறையில் வாழ்ந்த இனக்குழச் சமூகம் சங்க மருவிய காலத்தில் நிலவுமைமை சமூகமாக மாறியது தான். இரும்பின் பயன்பாடுகளுள் ஒன்றான ஆயுதங்களை உபயோகித்து தன்னுடைய நிலப்பரப்பிடை வுடைத்து அதற்குத் தான் ஒருவனே தலைவனாக விரும்பி பேராசைப் பட்டதன் விளைவே நிலவுடைமை சமூகமாக மலர்ந்தது. தனிமனித சமூகத்தில் சீர்கேடுகள் தோன்றுவதற்குாிய காரணமாக அமைந்தது. அவ்வகையில் குடிமக்களுடைய ஒழுக்கத்தை வலியுறுத்திக் கூறும் இவ்விலக்கியங்களை அரசும் அதிகாரம் படைத்தவர்களும் செல்வந்தர்களும் ஆதாத்தமையால் அற இலக்கியங்கள் வளர்ந்தன எனலாம்.

வள்ளுவர் கூறும் மனித நேயம்

உலகில் விவிலியத்திற்கு அடுத்து அதிக மொழிகளில் மொழிபெயர்க்கப்பட்டுள்ள நூல் திருக்குறள். சாதி மதம் இனம் மொழி நாடு எனும் வேறுபாடுகளுக்கு இடம்கொடாமல்

“யாதும் ஊரே யாவரும் கேளீர்” என உலக மாந்தர் அனைவரையும் ஒன்று சேர்க்கும் உன்னதமான இலக்கியம்.

"பிறப்பொக்கும் எல்லா உயிருக்கும் சிறப்பொவ்வா செய்

தொழில் வேற்றுமை யான்"

என்ற திருவள்ளுவரின் வாக்கிற்கேற்ப ஒருவன் செய்கிற செயல்களால் தான் பெருமையும் சிறுமையும் அவமை சென்றடையும் என்றும் பிறப்பினால்

உயர்வு தாழ்வு கிடையாது என்றும்

“அன்புடையார் எல்லாம் தமக்குரியர் அன்புடையார்

என்பும் உரியர் பிறார்கு 
என்று எடுத்த எடுப்பிலேயே மனித நேயத்துக்கு அடிப்படையாக அமைவது அன்பே என்று அழுத்தமாக கூறுகிறார். மக்களின் பாகுபாடுகள்

மக்கள் அனைவரும் பிறப்பால் ஒப்பார்இ தொழிலால் மாறுபடுவர் என்பது குறளின் பொதுக்கருத்து. இதனையே கவிமணி

“பிறப்பினால் எவர்க்கும் பெருமை வாராதப்பா சிறப்பு வேண்டுமெனில் நல்ல செய்கை வேண்டும்" என்பார். காலப்போக்கில் தொழிலால் மக்களுக்குள் பாகுபாடுகள் ஏற்பட்டன.

பிறப்பும் இறப்பும்

மனிதன் காலையில் விழித்தெழுந்து கடமைச் செய்துஇ உறங்கி ஓய்வு கொள்கிறான். அது போல இவ்வுலகில் மனிதனுடைய பிறப்பும் உறங்கி விழிப்பது போலவும் மீண்டிம்

"உறங்கி ஓய்வு கொள்வது இறப்பது போலவும்

விழிப்பது போலவும் பிறப்பு" (குறள்: 334)

என்னும் குறளால் காட்டுகிறார் வள்ளுவப் பெருமகனார்.

அறம் பற்றி தனிமனிதனுக்கு உரைப்பவை

அறம் என்னும் சொல் தானம் தர்மம் மட்டுமன்றி பிறர்க்குப் பயன் கருதாது செய்யும் அனைத்து நற்காரியங்களையும் குறிக்கும் சொல். அவ்வகையில் முதலில் நிற்பது பசிப்பிணிப் போக்குவது என மணிமேகலை கூறுகிறது.

"பசிப்பினி யென்னும் பாவியது தீர்ந்தோர்

இசைச்சொ லளவைக் கென்னா நிமிராது"

(மணி 81-84)

வறியவர்க்குக் காணிக்கை இடலும், யாகம் செய்தலும், தவம் செய்தலும் அறமாகும்.

செல்வத்துப் பயனே ஈதல் என்கிறது புறநானனூறு. ஒழுக்கம் என்னும் வாழ்வியல் கூறு மற்றெல்லாவற்றைக் காட்டிலும் சிறந்ததாகக் கருதப்படுகிறது. கல்வியைக் காட்டிலும் ஒழுக்கம் சிறந்தது. “ஒதலிற் ிறந்தது ஒழுக்கம் உடைமை" என்னும் முதுமொழிக்காஞ்சி வரி ஒழுக்கத்தின் மேன்மையை இயம்புகிறது. ஒழுக்கத்துடன் வாழ்வோரைச் சான்றோர் என்று சொல்லலாம்.

நோயும் மனித நேயமும்

“நோய்நாடி நோய்முதல் நாடிஅது தணிக்கும்

வாய்நாடி வாய்ப்பச் செயல்" ～～(திருக்குறள் - 948)

என்ற குறள் மூலம் வள்ளுவர் நோய் இன்னதென்று ஆராய்ந்து அந்நோய்க்கான காரணம் ஆராய்ந்து அதைக் குணப்படுத்தும் வழியையும் ஆராய்ந்து சிகிச்சையை மேற்கொள்ள வேண்டும் என்று நோயின் தன்மையைப் பற்றிக் கூறுகிறார்.

நோய் கண்ட மனிதனைச் சுகாதாரம் கருதிச் சக மனிதர்களிடமிருந்து பிரித்து வைக்கிறது மருத்துவ அறிவியல். இப்பிரிவு உடலால் மட்டும் இருக்கட்டும், உள்ளத்தால் வேண்டாம். உள்ளப்பிரிவு அவர்களின் உயிருக்கு ஊறு விளைவித்து விடும். ஆகையால் உயிர் சுமந்து திரியும் இந்நோய்வாய்ப்பட்டவர்களுக்கு உயிர்வாழ ஊக்கம் கொடுத்து உடற்குறையைத் தீர்க்க வேண்டும் என்று மனிதநேயமுள்ள பாடல் ஒன்றினை ஏலாதி கூறுகிறது.

“கருஞ்சிரங்கு வெண்தொழூ நோய் கல்வளி காயும்

பெருஞ்சிரங்கு பேர் வயிற்றுத் தீயார்க்கு- அருஞ்சிரமம்

ஆற்றுண் ஈத்து அவை தீர்த்தார் அரசராய்ப்

போற்றி ஊண் உண்பார் புரந்து" - (ஏலாதி - 57)

என்ற பாடலின் மூலம் கொடிய சிரங்கு நோயும், வெண்ணாறக் குட்ட நோயும், கல்லடைப்பு

நோயும், வாதநோயும், வருந்துகின்ற கழலை நோயும், பெரிய வயிற்றெரிச்சல் நோயுமாகிய இவற்றை உடையோர்க்கு உணவு,மருந்து ஆகியவை கொடுத்து அவர்கள் உற்ற நோயைத் தீர்க்க வேண்டும் என்பது மனிதநேயத்தை வெளிப்படுத்துகிறது.

கல்வி 
ஒருவன் எழுமையும் ஏமாப்பும் வாழ வழி செய்வது கல்வி. அறியாமை இருளை அகற்றவல்லது கல்வி. இத்தகைய உயர்ந்த கல்வியை ஒருவன் கட்டாயம் கற்க வேண்டும் என்றும் அவனுடைய பொருளாதார நிலை தாழ்ந்திருந்த போதிலும் அவன் பிச்சை ஏற்றேனும் கல்வியைக் கற்க வேண்டும்.

“பிச்சை புக்காயினுங் கற்றல் மிக இனிதே" (இனியவை - 1)

“உற்றுழி உதவியும் உறுபொருள் கொடுத்தும் பிற்றைநிலை முனியாது कற்றல் நன்றே"

என்று கல்வியின் இன்றியமையாமையைப் புறநானூறு புகழ்கிறது .

\section{குழந்தைச் செல்வம்}

' ீீகம் இல்லா வீடும் சிறுபிள்ளை இல்லா வீடும் சிறக்காது.' என்பது பழமொழி.

இல்லற வாழ்வின் பெருமைக்கு அடையாளமாக இருப்பது குழந்தைச் செல்வம். இதனை,

“குழவி தளர்நடை காண்டல் இனிதே

அவர் மழலை கேட்டல் அமிழ்தினும் இனிதே" - (இனியவை நாற்பது -14)

என்று குறிப்பிடிகிறது. ஈகை, புகழ், கொடை முதலான பதினாறு செல்வங்களைப் பெற்றாலும் குழந்தைச்செல்வம் இல்லாத வாழ்க்கை இன்பம் பயக்காது. சங்க இலக்கியத்திலும் இதன் சிறப்பை புறம் - 188 ஆம் பாடல் மிக அழகாகப் பேசுகிறது.

\section{நீதி இலக்கியத்தின் இயல்புகள்}

படிப்போரை உணர்ச்சிப் பெருக்கால் கிளர்ச்சி அடையச் செய்யாது சிறந்த கருத்துக்களைக் கூறி அவர்களது வாழ்க்கையைச் செம்மைப்படுத்துவதை நோக்கமாகக் கொண்டு உணர்ச்சியைத் தூண்டாமல் அறிவைத் தூண்டுவதையேக் குறிக்கோளாகக் கொண்டது. கலையழகுக்கு முதன்மை தராமல் கருத்துக்கு முதலிடம் தருவது. இவ்வாறு மனப்பண்புகளை மேம்படுத்துவதே நீதி இலக்கியத்தின் தன்மையாகும்.

\section{தொகுப்புரை}

சங்ககாலந்தொட்டு அறம் கூறப்பட்டு வந்தாலும் தனித்த நிலையில் சங்க மருவிய காலத்தில்தான் அறம் வலியுறுத்தப்பட்டது. மனிதன் தனித்தன்மையுடன் சிறப்புற்று வாழ தனிமனித ஒழுக்கமே முதன்மையானதாக அமைகிறது. மக்கள் மகிழ்ச்சி உடல்நலத்தைப் பொறுத்தது நோய் இன்றி வாழ்வதே எல்லாவற்றிலும் சிறப்பானது என்பதையும் குறள் வலியுறுத்தத் தவறவில்லை. நமது வாழ்க்கைமுறை சிறப்பானதாகவும், பெருமைமிக்கதாகவும், பிறரால் பாராட்டப்படக் கூடியதாகவும் அமையவேண்டுமெனில் நீதிநூல்கள் கூறும் அறக்கருத்துக்களை மனதில் கொண்டு அவற்றை தம் வாழ்வில் கடைபிடித்தால் சமூகத்தில் சிறந்து விளங்கி வாழ்வில் உன்னத நிலைக்கு மனிதனை உயர்த்துவதே அறநூல்களாகும். எனவே அறநூல்களின் பெருமை உணர்வோம் உலகிற்கும் உணர்த்துவோம்.

\section{பார்வை நூல்கள்}

1. தமிழ் இலக்கியங்களில் அறம் நீதி முறைமை பேரா.நா.சுப்புரெட்டியார்

ஐந்திணை பதிப்பகம், சென்னை - 05 டிசம்பர் 1988

2. திருக்குறள் பரிமேலழகர் மூலமும் உரையும்

3. புதிய நோக்கில் தமிழ் இலக்கிய வரலாறு - முனைவர் தமிழண்ணல்

4. பதினெண் கீழ்க்கணக்கு நூல்கள் தி.சு.பாலசுந்தரம் (உ.ஆ) கழக வெளியீடு) சென்னை - 18 (2004) 